\title{
Multiparametric 18F-FDG PET/MR follow-up crossuak in a patient with autoimmune pancreatitis
}

\author{
Isabel Rauscher*, Matthias Eiber, Hana Algül, Jens T. Siveke, Gregor Weirich, Anna M. Schlitter and Ambros J. Beer
}

\author{
* Correspondence: \\ isabel.rauscher@tum.de \\ Technical University of Munich \\ Klinikum rechts der Isar, Department \\ of Nuclear Medicine, Munich, \\ Germany
}

\begin{abstract}
Background: Positron emission tomography/magnetic resonance imaging (PET/MR) is a new multimodal imaging technique, which might improve the diagnostic performance not only in oncological patients but also in patients with non-neoplastic inflammatory lesions as routinely used ${ }^{18} \mathrm{~F}-\mathrm{FDG}$ is not a cancer specific agent.

Case Presentation: Multiparametric ${ }^{18}$ F-FDG PET/MR in a woman with pain in the upper abdomen and inconclusive laboratory and clinical data presenting with moderately increased, diffuse ${ }^{18} \mathrm{~F}-\mathrm{FDG}$ uptake with delayed contrast enhancement, diffusion-restriction and focal enlargement in the pancreatic body being suggestive for autoimmune pancreatitis (AIP). Follow-up ${ }^{18} \mathrm{~F}$-FDG PET/MR after initiation of steroid therapy confirmed complete resolution of imaging abnormalities.

Conclusion: ${ }^{18} \mathrm{~F}-\mathrm{FDG}$ PET/MR might be valuable in the management of AIP providing complementary data regarding accurate diagnosis and monitoring therapy response to avoid unnecessary surgery.
\end{abstract}

\section{Background}

Recently, positron emission tomography/magnetic resonance imaging (PET/MR) has been introduced as a new multimodal imaging technique combining morphological and functional information. For the diagnosis of pancreatic cancer, fluorine-18 fluorodeoxyglucose ( $\left.{ }^{18} \mathrm{~F}-\mathrm{FDG}\right)$-PET has been most widely used (Rijkers et al., 2014). However, ${ }^{18} \mathrm{~F}-\mathrm{FDG}$ is not a cancer specific agent and its uptake has been described in non-neoplastic inflammatory lesions as well (Ozaki et al., 2008).

\section{Case Presentation}

We present the case of a 58-year-old female with pain in the left upper abdomen since 2 months in the absence of other clinical symptoms. Laboratory data were inconclusive and a history of alcohol abuse could be excluded. Endoscopic ultrasound and CT ruled out choledocholithiasis, but revealed a bulky pancreas indicating inflammation. ${ }^{18}$ F-FDG PET/MR was performed to rule out possible unrecognized pancreatic cancer (Fig. 1). The scan demonstrated moderately increased, diffuse ${ }^{18}$ F-FDG uptake in the pancreatic body (A, arrow) with corresponding MRI showing enlargement of the pancreatic body with delayed contrast enhancement in T1 VIBE sequence (B, arrow), high diffusion-weighted imaging (DWI) signal $(C$; b value $=400)$ and low ADC values (D) being suggestive for autoimmune pancreatitis (AIP) as described before (Kamisawa et al.,

(c) The Author(s). 2017 Open Access This article is distributed under the terms of the Creative Commons Attribution 4.0 International License (http://creativecommons.org/licenses/by/4.0/), which permits unrestricted use, distribution, and reproduction in any medium, provided you give appropriate credit to the original author(s) and the source, provide a link to the Creative Commons license, and indicate if changes were made. 


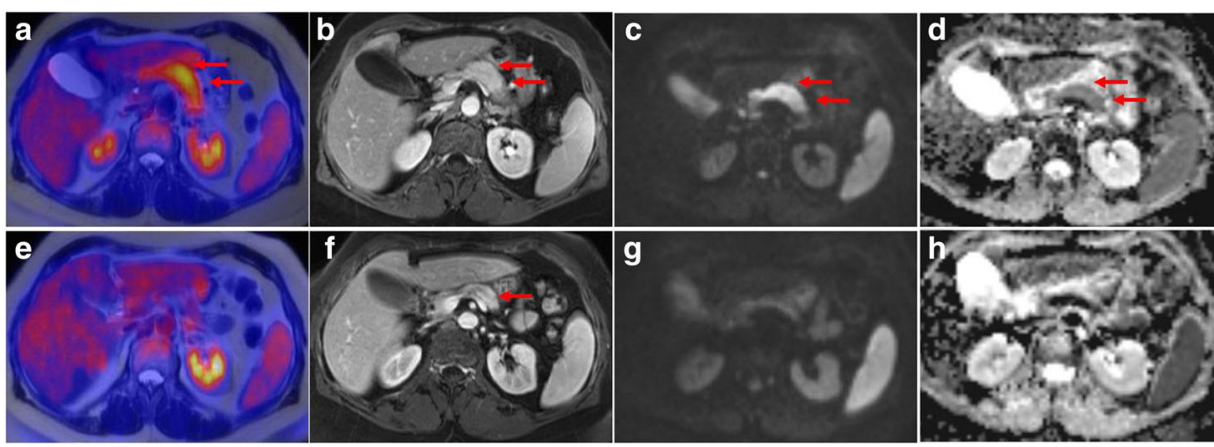

Fig. 1 Multiparametric ${ }^{18} \mathrm{~F}$-FDG PET/MR in a patient with autoimmune pancreatitis and follow-up ${ }^{18} \mathrm{~F}-\mathrm{FDG}$ PET/MR after initiation of steroid therapy

2010; Lee \& Sahani, 2014). Subsequently endoscopic ultrasound-guided biopsy of the pancreas excluded pancreatic cancer and confirmed chronic pancreatic inflammation. In general, AIP is responsive to corticosteroid therapy (Lee \& Sahani, 2014; Kamisawa et al, 2009). Follow-up ${ }^{18}$ F-FDG PET/MR 4 weeks after initiation of steroid therapy confirmed complete resolution of imaging abnormalities with normalization of ${ }^{18} \mathrm{~F}-\mathrm{FDG}$ uptake (E), size reduction of affected pancreatic parenchyma with normalization of pancreatic enhancement $(\mathrm{F})$ and diffusion restriction $(\mathrm{G}$ and $\mathrm{H})$.

\section{Discussion}

Along with clinical, laboratory, and histopathological data, imaging plays an important role in the diagnosis and management of AIP, however, imaging appearance of AIP varies widely. Certain nonspecific imaging features in cross-sectional imaging have been defined which are more likely to represent AIP than alternate diagnoses (e.g. pancreatic cancer) (Lee \& Sahani, 2014). Recent studies suggest that ${ }^{18} \mathrm{~F}$-FDG PET may prove valuable in providing complementary data in delineating the extent of organ involvement and staging the extent of disease as extra-pancreatic sites of involvement are also often seen in AIP (Lee \& Sahani, 2014). Further, additional ${ }^{18} \mathrm{~F}$-FDG PET might help guiding biopsy early in the diagnostic evaluation, and monitoring response to therapy.

\section{Conclusion}

In complex cases, when it's important to gather all available functional and morphological information to exclude malignancy, combined ${ }^{18} \mathrm{~F}-\mathrm{FDG}$ PET/MR may prove valuable in the management of AIP regarding accurate diagnosis and monitoring therapy response to avoid unnecessary surgery.

Acknowledgements

The publication of this article was supported by funds of the European Association of Nuclear Medicine (EANM).

Funding

No funding was received for this study. 
Ethics approval and consent to participate

For this case report, informed written consent for publication was obtained from the patient. As the FDG-PET/CT was part of a routinely performed clinical examination no ethics approval was needed.

\section{Competing interests}

The authors declare that they have no conflicts of interest or competing interests.

\section{Publisher's Note}

Springer Nature remains neutral with regard to jurisdictional claims in published maps and institutional affiliations.

Received: 12 July 2017 Accepted: 23 October 2017

Published online: 22 November 2017

\section{References}

Rijkers AP, Valkema R, Duivenvoorden HJ et al (2014) Usefulness of F-18-fluorodeoxyglucose positron emission tomography to confirm suspected pancreatic cancer: a meta-analysis. Eur J Surg Oncol 40:794-804

Ozaki Y, Oguchi K, Hamano H et al (2008) Differentiation of autoimmune pancreatitis from suspected pancreatic cancer by fluorine-18 fluorodeoxyglucose positron emission tomography. J Gastroenterol 43:144-151

Kamisawa T, Takuma K, Anjiki H et al (2010) Differentiation of autoimmune pancreatitis from pancreatic cancer by diffusion-weighted MRI. Am J Gastroenterol 105:1870-1875

Lee LK, Sahani DV (2014) Autoimmune pancreatitis in the context of IgG4-related disease: review of imaging findings. World J Gastroenterol 20:15177-15189

Kamisawa T, Shimosegawa T, Okazaki K et al (2009) Standard steroid treatment for autoimmune pancreatitis. Gut 58 : $1504-1507$

\section{Submit your manuscript to a SpringerOpen ${ }^{\circ}$} journal and benefit from:

- Convenient online submission

- Rigorous peer review

- Open access: articles freely available online

- High visibility within the field

- Retaining the copyright to your article

Submit your next manuscript at $\gg$ springeropen.com 